\title{
SARS-CoV-2 infection in dialysis and kidney transplant patients: immunological and serological response
}

\author{
Federico Alberici ${ }^{1,2}\left(\right.$ - Stefania Affatato ${ }^{1,2}$. Daniele Moratto ${ }^{3}$. Federica Mescia ${ }^{1,2}$ - Elisa Delbarba ${ }^{1,2}$. \\ Alice Guerini ${ }^{1,2} \cdot$ Martina Tedesco $^{1,2} \cdot$ Peter D. Burbelo ${ }^{4} \cdot$ Roberta Zani $^{1,2} \cdot$ Ilaria Castagna ${ }^{1,2} \cdot$ Agnese Gallico $^{1,2}$. \\ Mattia Tonoli ${ }^{1,2}$. Margherita Venturini ${ }^{1,2}$. Aldo M. Roccaro ${ }^{5}$. Mauro Giacomelli, ${ }^{6,7}$. Jeffrey I. Cohen ${ }^{8}$. \\ Viviana Giustini $^{3,5} \cdot$ Kerry Dobbs $^{9} \cdot$ Helen C. Su $^{9}$. Chiara Fiorini ${ }^{10} \cdot$ Virginia Quaresima $^{10} \cdot$ Fabio Battista Viola $^{1,2}$. \\ Valerio Vizzardi ${ }^{1,2}$ - Mario Gaggiotti ${ }^{1,2}$. Nicola Bossini ${ }^{1,2}$. Paola Gaggia ${ }^{1,2}$ - Raffaele Badolato ${ }^{6,7}$. \\ Luigi D. Notarangelo $0^{9} \cdot$ Marco Chiarini $^{3} \cdot$ Francesco Scolari $^{1,2}$
}

Received: 17 August 2021 / Accepted: 15 November 2021 / Published online: 24 January 2022

(c) The Author(s) under exclusive licence to Italian Society of Nephrology 2021

\begin{abstract}
Background Dialysis and kidney transplant patients with moderate-severe COVID-19 have a high mortality rate, around 30\%, that is similar in the two populations, despite differences in their baseline characteristics. In these groups, the immunology of the disease has been poorly explored.

Methods Thirty-two patients on dialysis or with kidney transplant and SARS-CoV-2 infection requiring hospitalization (COV group) were included in our study. Lymphocyte subsets, dendritic cell (DC) counts and monocyte activation were studied. SARS-CoV-2 anti-spike/anti-nucleocapsid were monitored, and baseline cytokines and chemokines were measured in 10 patients.

Results The COV group, compared to healthy subjects and uninfected dialysis/kidney transplant controls, showed lower numbers of CD4 + and CD8 + T cells, Natural-Killer (NK), B cells, plasmacytoid and myeloid DCs, while the proportion of terminally differentiated B-cells was increased. IL6, IL10, IFN- $\alpha$ and chemokines involved in monocyte and neutrophil recruitment were higher in the COV group, compared to uninfected dialysis/kidney transplant controls.

Patients with severe disease had lower CD4 +, CD8 + and B-cell counts and lower monocyte HLA-DR expression. Of note, when comparing dialysis and kidney transplant patients with COVID-19, the latter group presented lower NK and pDC
\end{abstract}

Federico Alberici, Stefania Affatato and Daniele Moratto joint first authors.

Raffaele Badolato, Luigi D. Notarangelo, Marco Chiarini and Francesco Scolari joint last authors.

Federico Alberici

federico.alberici@unibs.it

1 Department of Medical and Surgical Specialties, Radiological Sciences and Public Health, University of Brescia, Brescia, Italy

2 Nephrology Unit, Spedali Civili Hospital, ASST Spedali Civili di Brescia, Piazzale Spedali Civili 1, 25125 Brescia, Italy

3 Flow Cytometry Unit, Clinical Chemistry Laboratory, ASST Spedali Civili di Brescia, Brescia, Italy

4 National Institute of Dental and Craniofacial Research, National Institutes of Health, Bethesda, MD, USA

5 Clinical Research Development and Phase I Unit, ASST Spedali Civili di Brescia, Brescia, Italy
6 Department of Clinical and Experimental Sciences, University of Brescia, Brescia, Italy

7 “Angelo Nocivelli” Institute of Molecular Medicine, University of Brescia, ASST Spedali Civili, Brescia, Italy

8 Laboratory of Infectious Diseases, National Institute of Allergy and Infectious Diseases, National Institutes of Health, Bethesda, MD, USA

9 Laboratory of Clinical Immunology and Microbiology, National Institute of Allergy and Infectious Diseases, National Institutes of Health, Bethesda, MD, USA

10 Centro di Ricerca Emato-Oncologica AIL (CREA), Diagnostic Department, ASST Spedali Civili di Brescia, Brescia, Italy 
counts and monocyte HLA-DR expression. Up to 60 days after symptom onset, kidney transplant recipients showed lower levels of anti-spike antibodies compared to dialysis patients.

Conclusions During SARS-CoV-2 infection, dialysis and kidney transplant patients manifest immunophenotype abnormalities; these are similar in the two groups, however kidney transplant recipients show more profound alterations of the innate immune system and lower anti-spike antibody response.

\section{Graphical abstract}

\section{SARS-COV-2 INFECTION IN DIALYSIS AND KIDNEY TRANSPLANT PATIENTS: IMMUNOLOGICAL AND SEROLOGICAL RESPONSE}

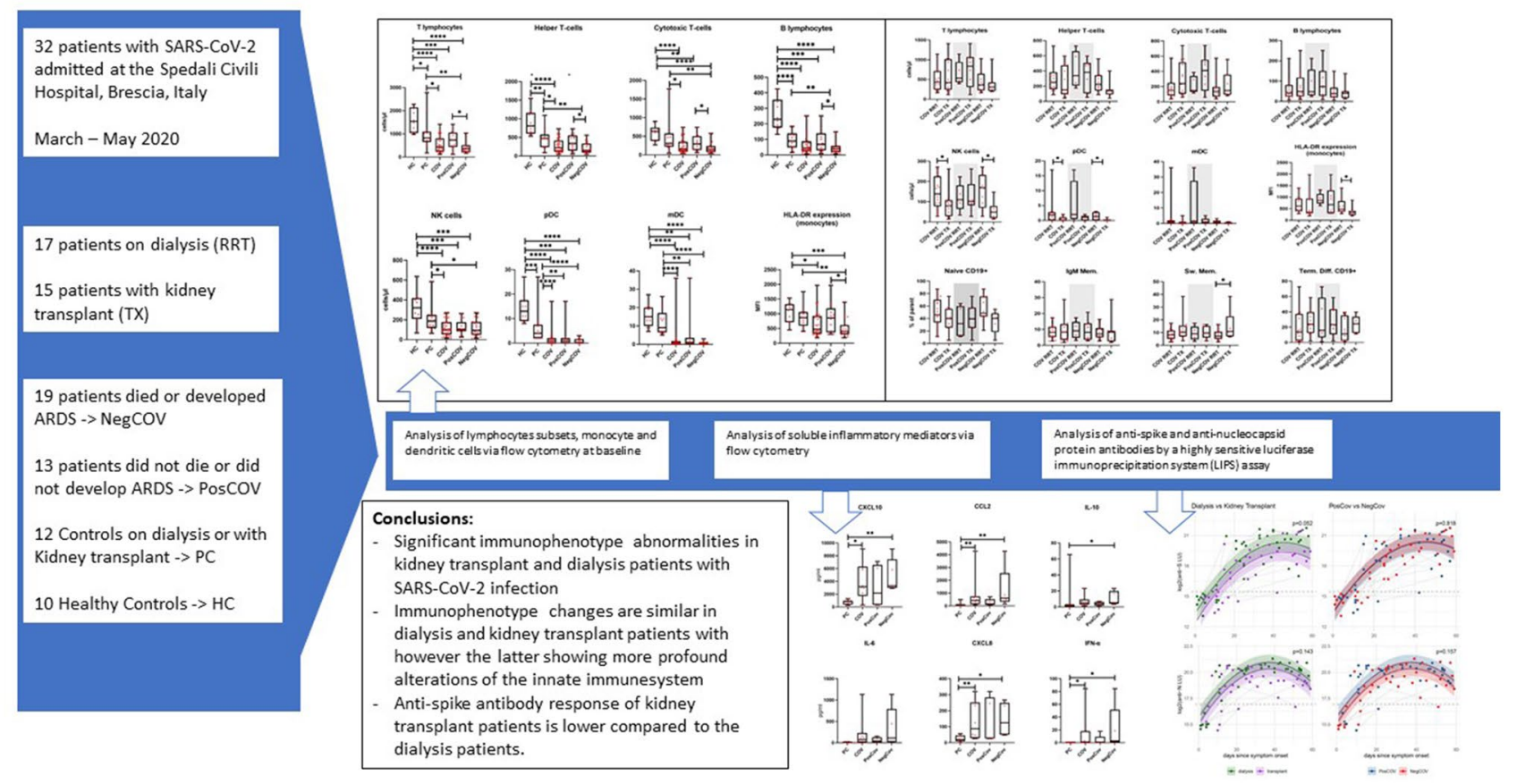

Keywords Hemodialysis $\cdot$ Kidney transplant $\cdot$ Lymphocytes $\cdot$ SARS-CoV-2 $\cdot$ COVID-19

\section{Introduction}

SARS-CoV-2 infection is a medical emergency posing challenges worldwide. Prognostic factors for poor outcome from acute coronavirus 2019 disease (COVID-19) in the general population include age and comorbidities, such as hypertension and diabetes [1]. Several biomarkers of COVID-19 as well as of its severity have been identified [2-5], including elevation in neutrophil and monocyte counts, lymphopenia, $\mathrm{CD} 4+$ and $\mathrm{CD} 8+\mathrm{T}$ cell reduction as well as relative increase in activated CD4 + and CD8 + T cells. Despite conflicting reports on circulating B cells in COVID-19 [6, 7], the production of specific antibodies has been consistently documented as early as 7-14 days from the onset of symptoms $[6,8]$. Other biomarkers of disease severity in patients with COVID-19 include elevated levels of IL6, IL8, IFN- $\alpha$, IL18, CXCL10, IL10 and TNF- $\alpha$ [9-11] and a severe reduction of circulating plasmacytoid dendritic cells (pDCs) [12].
Despite their intrinsically higher frailty, dialysis and kidney transplant patients show variable clinical course and outcomes of SARS-CoV-2 infection, ranging from asymptomatic to aggressive forms [13, 14]. Overall, the fatality rate is similar in both groups (around 30\%) and higher compared to age-matched patients in the general population, supporting the idea that chronic kidney disease acts as a shared risk factor for severe COVID-19 [13-17].

Patients on dialysis and those with kidney transplant are well known to have abnormalities in the innate and adaptive immune system [18]. During end-stage renal disease (ESRD), the thymus undergoes involution, leading to naïve $\mathrm{T}$ cell lymphopenia and expansion of highly differentiated effector T cells [19]; of note, these abnormalities persist even after kidney transplant [20]. Some studies have reported that B cell lymphopenia can also occur in this context [21], especially of memory B cells [22]. Moreover, lower numbers of circulating natural-killer (NK) and dendritic cells (DCs), and reduced effector T-cell response, all 
implicated in the immune response against viruses, have also been described [18]. Based on these studies, it has been estimated that patients with ESRD have an immunological age that is approximately 20 years older than their chronological age [19].

Data on lymphocyte subsets in COVID-19 in ESRD and kidney transplant patients are scanty. One small study reported the immunological features of 7 hemodialysis patients affected by COVID-19. In this population, the percentage of activated T-cells $(\mathrm{CD} 3+\mathrm{HLA}-\mathrm{DR}+)$ was increased; moreover, lower levels of NK cells were observed in both hemodialysis and non-hemodialysis patients with COVID-19 compared to healthy controls [23]. A correlation between the degree of lymphopenia and COVID-19 severity and outcome has been described in kidney transplant recipients [13]. Furthermore, a study in 18 kidney transplant recipients with COVID-19 revealed an increased proportion of activated $\mathrm{B}$ cells and reduced numbers of memory CD4 + and CD8 + T-cells, but no differences in exhausted CD4 + and CD8 + T cells, compared to kidney transplant controls without COVID-19 [24].

We therefore aimed to examine the immunophenotype of dialysis and kidney transplant patients with SARS-CoV-2 infection requiring hospital admission, and to identify immunological abnormalities that may serve as biomarkers and possible predictors of disease severity in this context. Furthermore, we looked for possible differences between dialysis and kidney transplant patients by comparing their immunophenotyping features at the time of admission for COVID-19. Finally, in order to improve our understanding of the immunological changes during COVID-19 in these patients, we also measured plasma levels of soluble biomarkers and of SARS-CoV-2-specific antibodies during follow-up.

\section{Patients and methods}

We studied 32 patients on dialysis $(n=17)$ or recipients of kidney transplant ( $n=15$ ) with COVID-19 (COV cohort) who were admitted to Spedali Civili di Brescia Hospital from March 18 to May 22, 2020. The diagnosis of SARSCoV-2 infection (COV cohort) was based on a positive reverse transcription polymerase chain reaction test (RTPCR) on nasopharyngeal swab. Twelve -age-matched dialysis or kidney transplant recipients without SARS-CoV-2 infection, and 10 healthy adults (mean age 38 years, range 27-55) were included as pathological controls (PCs) and healthy controls ( $\mathrm{HCs}$ ), respectively. $\mathrm{COV}$ patients were further categorized into patients with a benign disease course (PosCOV), who did not die nor develop ARDS (defined as per the Berlin criteria [25]), and patients with an aggressive disease course (NegCOV), who developed ARDS and/or died.

Patients were treated according to the protocol in use at our center during the first wave of COVID-19 [26]. Briefly, antiviral therapy with lopinavir/ritonavir associated with hydroxychloroquine (dose-adjusted according to kidney function) was considered for all patients, unless contraindicated. Patients received this combination therapy for a minimum of 7 days and up to a maximum of 15 days. In case of shortage of lopinavir/ritonavir, darunavir and ritonavir were administered. Patients experiencing clinical deterioration after at least 7 days following symptom onset, with escalating oxygen requirements, progression of pulmonary disease based on chest X-ray, and no signs of bacterial infection, were considered for dexamethasone $(20 \mathrm{mg} /$ daily for 5 days, then $10 \mathrm{mg} /$ daily for 5 days) and up to two tocilizumab infusions at $12-24 \mathrm{~h}$ intervals $(8 \mathrm{mg} / \mathrm{kg}$ of body weight, maximum dose per infusion: $800 \mathrm{mg}$ ).

Blood samples were collected from all patients at baseline (within up to 2 days after admission) in order to perform immunophenotyping. In hemodialysis patients, samples were collected before the first dialysis session performed during admission. For a subgroup of 13 patients, at least one further longitudinal sample for immunophenotyping was collected. Plasma levels of soluble inflammatory mediators were measured at baseline in 10 patients. Both the analyses mentioned above were performed in selected sub-cohorts of patients, that were balanced by design in terms of number of dialysis/kidney transplant patients, as well as disease severity (PosCov/NegCov).

Levels of anti-spike (anti-S) and anti-nucleocapsid (anti-N) IgG antibodies were measured in all patients at baseline and monitored for up to 2 months from symptom onset.

Ethical approval (protocol NP 4027, date 03/23/2020) for this study was obtained according to Italian regulations; all patients signed informed consent.

\section{Flow cytometric analysis of lymphocyte subsets, monocytes and dendritic cells}

Flow cytometric analyses were all performed on fresh peripheral whole blood samples of patients and controls; cells were stained according to the manufacturer's protocols by using appropriate mixtures of monoclonal antibodies (MoAbs; all from BD Bioscience).

A combination of monoclonal antibodies (MoAbs) directed against CD3 (UCHT1), CD4 (RPA-T4), CD8 (SK1), CD16 (3G8), CD56 (NCAM16.2), CD19 (SJ25C1), HLADR (G46-6), and CD45 (2D1) was used for quantification of the main lymphocyte populations: CD3 + total lymphocytes, $\mathrm{CD} 3+\mathrm{CD} 4+$ helper and CD3 + CD8 + cytotoxic T cells, 
CD4 + HLA-DR + and CD8 + HLA-DR + activated T cells, CD19+B lymphocytes, and CD3-CD56/16 + NK cells.

A combination of MoAbs directed against CD45RA (HI100), CCR7 (150,503), CXCR5 (RF8B2), CD31 (WM59), CD3 (UCHT1), CD4 (RPA-T4), and CD8 (SK1) was used to evaluate T-lymphocyte subsets: CD4+CD45 $\mathrm{RA}+\mathrm{CCR} 7+\mathrm{CD} 31+$ recent thymic emigrants, CD4 + and
CD $8+$ CD45RA + CCR7 + naïve cells, CD4 + and CD8 + CD45RA + CCR7- terminally differentiated cells, and CD4 + CD45RA-CCR7 + CXCR5 + follicular T cells.

A combination of MoAbs directed against CD10 (HI10a), CD19 (SJ25C1), CD20 (L27), CD21 (B-ly4), CD27 (L128), sIgD (IA6-2), sIgM (G20-127), and CD38 (HIT2) was used to evaluate B lymphocyte subsets:
Table 1 Baseline clinical characteristics in 32 dialysis and kidney transplant patients with SARS-CoV-2 infection

\begin{tabular}{|c|c|c|c|}
\hline & PosCOV [13] & NegCOV [19] & $p$ \\
\hline \multicolumn{4}{|l|}{ Characteristics } \\
\hline Age (years) & $57(48-73)$ & $73(60-83)$ & 0.035 \\
\hline Male/female & $13 / 0$ & $13 / 6$ & 0.035 \\
\hline Dialysis/Kidney transplant patients & $5 / 8$ & $12 / 7$ & 0.280 \\
\hline \multicolumn{4}{|l|}{ Comorbidities } \\
\hline Hypertension & $12 / 13$ & $16 / 18$ & 0.766 \\
\hline Diabetes & $3 / 13$ & $6 / 18$ & 0.826 \\
\hline Obesity & $2 / 13$ & $6 / 18$ & 0.477 \\
\hline \multicolumn{4}{|l|}{ Symptoms at onset } \\
\hline Temperature & $9 / 13$ & $18 / 19$ & 0.132 \\
\hline Cough & $7 / 13$ & $2 / 19$ & 0.015 \\
\hline Shortness of breath & $3 / 13$ & $6 / 19$ & 0.704 \\
\hline \multicolumn{4}{|l|}{ Baseline Chest X-Ray } \\
\hline No infiltrates & $0 / 13$ & $1 / 15$ & 1 \\
\hline Unilateral infiltrates & $1 / 13$ & $1 / 15$ & 1 \\
\hline Bilateral infiltrates & $12 / 13$ & $13 / 15$ & 1 \\
\hline 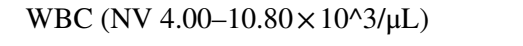 & $7.045(4.35-7.61)$ & $6.240(3.93-8.49)$ & 0.668 \\
\hline Lymphocytes (NV $\left.0.90-4.00 \times 10^{\wedge} 3 / \mu \mathrm{L}\right)$ & $0.74(0.51-1.4)$ & $0.43(0.36-1.02)$ & 0.026 \\
\hline Neutrophils (NV $1.50-8.00 \times 10^{\wedge} 3 / \mu \mathrm{L}$ ) & $5.04(2.93-6.34)$ & $4.89(3.06-7.75)$ & 0.451 \\
\hline Monocytes (NV $\left.0.2-1 \times 10^{\wedge} 3 / \mu \mathrm{L}\right)$ & $0.57(0.50-0.67)$ & $0.41(0.30-0.47)$ & 0.003 \\
\hline Creatinine $(\mathrm{mg} / \mathrm{dL}) *$ & $1.8(1.3-3.8)$ & $3.3(1.6-6.1)$ & 0.209 \\
\hline $\mathrm{CRP}(\mathrm{NV}<5.0 \mathrm{mg} / \mathrm{L})$ & $49(37-108)$ & $113(46-1023)$ & 0.173 \\
\hline D-Dimer (NV 0-232 ng/mL) & $360(219-679)$ & $454(233-1192)$ & 0.538 \\
\hline Ferritin (ug/L) & $781(299-874)$ & $412(201-1023)$ & 0.473 \\
\hline Fibrinogen (mg/dL) & $614(416-747)$ & $492(384-643)$ & 0.220 \\
\hline \multicolumn{4}{|l|}{ Kidney transplant patients } \\
\hline Age of transplantation (years) & $10.0(2.0-18.0)$ & $8.5(4.3-20.0)$ & 0.731 \\
\hline \multicolumn{4}{|l|}{ Induction regimen } \\
\hline Thymoglobuline & $3 / 8$ & $3 / 7$ & 0.681 \\
\hline Basiliximab & $2 / 8$ & $3 / 7$ & 0.464 \\
\hline Alemtuzumab & $1 / 8$ & $1 / 7$ & 0.509 \\
\hline \multicolumn{4}{|l|}{ Baseline immunosuppression } \\
\hline $\mathrm{CNI}$ & $8 / 8$ & $6 / 7$ & 0.467 \\
\hline mTOR-I & $2 / 8$ & $0 / 7$ & 0.467 \\
\hline MMF & $5 / 8$ & $6 / 7$ & 0.569 \\
\hline Glucocorticoids & $7 / 8$ & $7 / 7$ & 1 \\
\hline Basal creatinine $(\mathrm{mg} / \mathrm{dL})$ & $1.0(1.0-1.8)$ & $2.5(1-3.8)$ & 0.097 \\
\hline
\end{tabular}

Patients were stratified according to the evolution of COVID-19 in a group with positive outcome (Pos$\mathrm{COV}$, patients that did not die or develop ARDS) and in a group with negative outcome (NegCOV, patients that died or developed ARDS). Data are reported as number for categorical variables and median (interquartile range) for continuous variables

$H D$ hemodialysis, $P D$ peritoneal dialysis, $W B C$ white blood cells, $N V$ normal value, $C R P$ c-reactive protein *Reported only for kidney transplant patients 
Table 2 Management of SARS-CoV-2 infection in 32 dialysis and kidney transplant patients

\begin{tabular}{llll}
\hline Characteristics & $\begin{array}{l}\text { Positive } \\
\text { outcome } \\
{[13]}\end{array}$ & $\begin{array}{l}\text { Negative } \\
\text { outcome } \\
{[19]}\end{array}$ & $p$ \\
\hline Patients on HD or PD & 5 & 12 & 0.280 \\
COVID-19 management & & & \\
Dexamethasone & $2 / 5$ & $8 / 12$ & 0.593 \\
Tocilizumab & $0 / 5$ & $1 / 12$ & 1 \\
Hydroxychloroquine & $5 / 5$ & $11 / 12$ & 1 \\
Antivirals* & $5 / 5$ & $12 / 12$ & 1 \\
Antibiotics & $5 / 5$ & $12 / 12$ & 1 \\
Kidney transplant patients & 8 & 7 & 0.280 \\
Immunosuppression management & & & \\
MMF, mTOR-I, CNI withdrawal & $8 / 8$ & $7 / 7$ & 1 \\
Glucocorticoids introduction or & $7 / 8$ & $8 / 8$ & 1 \\
$\quad$ increase & & & \\
COVID-19 Management & & & \\
Dexamethasone & $1 / 8$ & $4 / 7$ & 0.12 \\
Tocilizumab & $0 / 8$ & $4 / 7$ & 0.026 \\
Hydroxychloroquine & $8 / 8$ & $7 / 7$ & 1 \\
Antivirals* & $8 / 8$ & $7 / 7$ & 1 \\
Antibiotics & $4 / 8$ & $5 / 7$ & 0.608 \\
\hline
\end{tabular}

Patients were stratified according to the evolution of COVID-19 in a group with positive outcome (PosCOV, patients that did not die or develop ARDS) and in a group with negative outcome (NegCOV, patients that died or developed ARDS). Data are reported as number for categorical variables and median (interquartile range) for continuous variables

$H D$ hemodialysis, $P D$ peritoneal dialysis, $C N I$ calcineurin inhibitors, mTOR-I mammalian target of rapamycin inhibitors, $M M F$ mycophenolate mofetil

*Lopinavir/ritonavir, darunavir and ritonavir

CD19+CD20-CD27-IgD + naïve B cells, CD19+CD20CD27 + CD38hi terminally differentiated cells, $\mathrm{CD} 19+\mathrm{CD} 20+\mathrm{CD} 10-\mathrm{CD} 21+\mathrm{CD} 27+\operatorname{IgD}+\operatorname{IgM}+\operatorname{IgM}$ memory cells, and CD19+CD20+CD10$\mathrm{CD} 21+\mathrm{CD} 27 \pm \mathrm{IgD}-\mathrm{IgM}$ - switched memory cells.

A combination of MoAbs directed against CD45 (2D1), CD4 (RPA-T4), CD20 (L27), CD14 (M 99 ), CD123 (7G3), CD303 (V24-785), CD1c (F10/21A3), and HLA-DR (G46-6) was used for the quantification of CD45dimCD4dimCD123 + CD303 + plasmacytoid dendritic cells, and CD45dimCD4dimCD20-CD1c + myeloid dendritic cells, and for the evaluation of HLA-DR expression on CD45 + CD14 + CD4dim monocytes.

Stained samples were acquired on a Canto II (BD Bioscience) flow cytometer and analyzed using DIVA software version 8.0.2.

\section{Analysis of soluble inflammatory mediators}

Plasma levels of CXCL10 (IP-10), CXCL8 (IL8), CXCL9 (MIG), CCL5 (RANTES) and CCL2 (MCP-1) were analyzed by a flow cytometric bead array method, using the Human Chemokine Kit (Becton Dickinson, San Jose CA), following the manufacturer's instructions. Levels of IL1B, IL2, IL4, IL5, IL6, IL10, TNF- $\alpha$, IL17A, IFN- $\alpha$ and IFN- $\gamma$ were measured by the Flex set custom cytometric bead array technique (Becton Dickinson). Data were acquired on BD FACSCanto II flow-cytometer and analyzed by FCAP v3 software.

\section{Anti-SARS-CoV-2 IgG antibodies}

IgG antibodies to SARS-CoV-2 Spike (S) and Nucleocapside $(\mathrm{N})$ proteins were measured by a highly sensitive luciferase immunoprecipitation system (LIPS) assay, as described elsewhere [8]. Cutoff limits for determining positive $\mathrm{S}$ and $\mathrm{N}$ antibodies were based on the mean plus 3 and 4 standard deviations (SDs), respectively, of the serum values derived from 32 uninfected blood donor controls, and corresponded to 45,000 and 125,000 Light Units (LU), respectively.

\section{Statistical analyses}

Statistical analysis was performed using R software (https:// www.rproject.org) and GraphPad Prism 9. Results are expressed as the number and percentage for categorical variables and the median (interquartile range [IQR]) for continuous variables. Changes in variables were compared by unpaired or paired $t$-test, Wilcoxon test or Mann-Whitney test as appropriate; proportions of patients were compared using Fisher's test. Longitudinal changes in immunophenotyping parameters were analyzed fitting linear mixed models using the R package lmerTest [27]. Model predictors included time, COVID-19 outcome group and their interaction term as fixed effects, and patient ID as random effect. Longitudinal changes in anti-SARS-CoV-2 antibodies were also analyzed using linear mixed models with quadratic time trend, patient subgroup (dialysis/kidney transplant) and COVID-19 outcome group as fixed effects, and patient ID as random effect. Non-normally distributed variables were $\log$ transformed before fitting the models. $p<0.05$ (2-tailed) were considered significant. 

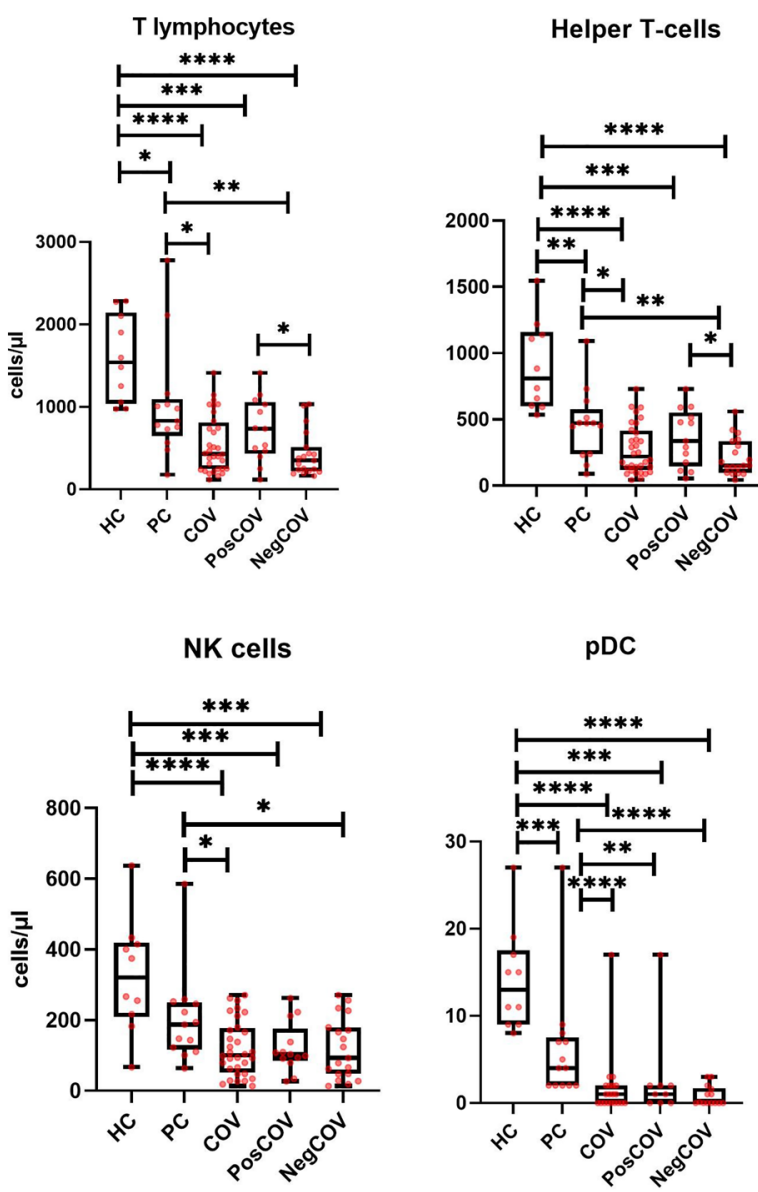

Fig. 1 Major lymphocyte subset and dendritic cell counts, and monocyte HLA-DR expression at baseline. Comparisons are made across five groups: healthy controls (HC), pathological controls on dialysis or with kidney transplant without SARS-CoV-2 infection (PC), the overall dialysis and kidney transplant population with SARS-CoV-2 infection (COV), the subgroup with SARS-CoV-2 infection and positive outcome (PosCOV) and the subgroup with SARS-CoV-2

\section{Results}

\section{Study population}

The main characteristics of the study population and management of SARS-CoV-2 infection are summarized in Tables 1, 2 and Supplementary Table 1. In brief, we included 17 dialysis patients (14 on hemodialysis and 3 on peritoneal dialysis) and 15 kidney transplant recipients, among whom 14 were on calcineurin inhibitors, 11 on mycophenolate mofetil and 13 on low dose glucocorticoids as baseline immunosuppression. Nineteen of the 32 patients $(59 \%)$ died or developed ARDS (NegCOV group). The 13 patients (41\%) who developed ARDS were equally distributed among the dialysis group and the kidney transplant group (6 vs. 7). Ten patients (31\%) died, most of whom belonged to the dialysis group (8 vs. 2 ).
Cytotoxic T-cells
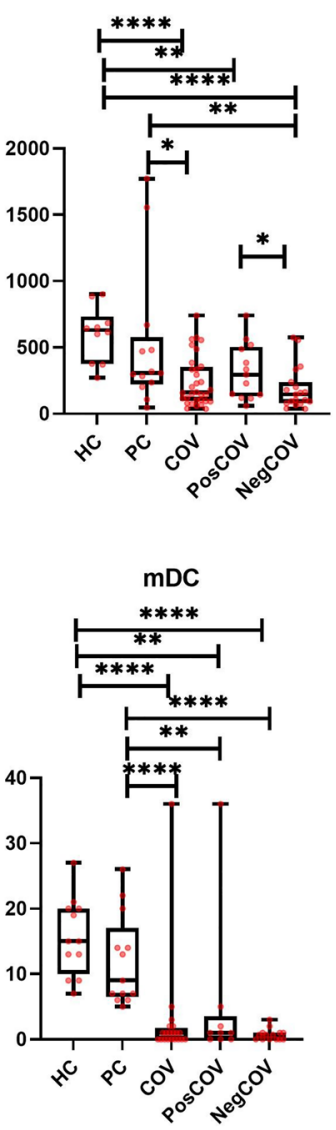

B lymphocytes

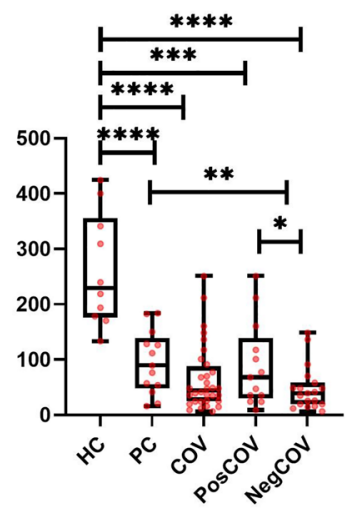

HLA-DR expression (monocytes)

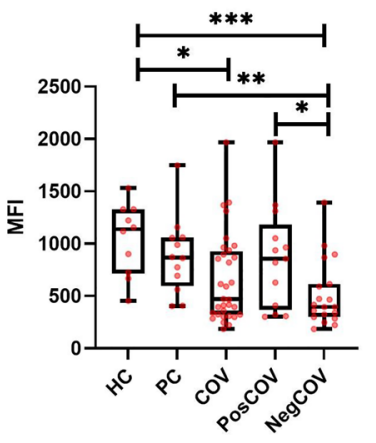

infection and negative outcome (NegCOV). The lines inside the boxes represent the median level, the edge of the boxes the 25th75th percentiles, the whiskers the minimum and maximal values. $M F I$ mean fluorescence intensity, $p D C$ plasmacytoid dendritic cells, $m D C$ myeloid dendritic cells. $* p<0.05, * * p<0.01, * * * p<0.001$, $* * * * p<0.0001$

NegCOV patients were older and showed lower lymphocyte and monocyte counts, compared to patients with a benign disease course (PosCOV group); a trend towards higher creatinine levels was also documented within the transplanted patients of the NegCOV group. Within the dialysis group, three patients were on peritoneal dialysis (PD). PD patients were younger than the other patients with COVID-19, and none of them developed ARDS or died (Supplementary Table 1).

\section{Flow cytometric analysis}

Immunophenotypic analysis at baseline revealed that the PC group showed, on average, lower numbers of all major lymphocyte and DC subsets compared to the HC group (Fig. 1). These cell counts were further reduced in the COV group. NegCOV patients displayed significantly 
lower counts of $\mathrm{CD} 3+, \mathrm{CD} 4+, \mathrm{CD} 8+$ and $\mathrm{B}$ cells compared to the PosCOV and PC groups. The number of NK, pDCs and $\mathrm{mDCs}$ were also significantly reduced in COV patients compared to the control groups, but no statistically significant differences were observed between levels in the PosCOV and NegCOV groups.

We also quantified monocyte HLA-DR expression, which was previously found to be markedly reduced in severe COVID-19 [12, 28, 29]. Our data confirmed a significant reduction in HLA-DR expression (measured as mean fluorescence intensity, [MFI]) in patients with a more aggressive disease course (NegCOV), compared to all other groups (Fig. 1).

All these immunophenotypic changes remained similar even after stratification of the overall population in the dialysis and kidney transplant subgroups (Supplementary Figs. 1 and 2). Notably, within the dialysis group, no significant differences were detected between hemodialysis and peritoneal dialysis patients, albeit in the context of very low statistical power due to small sample size, especially for the peritoneal dialysis group (Supplementary Fig. 3).

The proportion of $\mathrm{CD} 4+$ and $\mathrm{CD} 8+\mathrm{T}$ lymphocytes expressing HLA-DR was significantly increased in COV patients compared to the $\mathrm{HC}$ group, but values were similar to those observed in PCs (Fig. 2), indicating that SARSCoV-2 infection did not significantly change the activation status of circulating $\mathrm{T}$ lymphocytes, at least in the setting of our dialysis and kidney transplant cohorts assessed at a time point close to symptom onset. Likewise, COV patients displayed a distribution of CD8 + T-cell subsets (naïve, central memory, effector memory, terminally differentiated $\mathrm{CD} 8+\mathrm{T}$ cells) that was comparable to what was observed in the PC group (Fig. 2), despite being significantly more skewed towards terminally differentiated cells than in HCs. A similar pattern was observed for CD4 + T-cell subsets, that had overall similar distributions in the PC and COV groups (with only a statistically significant lower proportion of effector memory CD4+cells in COV), while the most marked differences were found when comparing $\mathrm{HCs}$ and PCs. No differences were detected between COV patients and $\mathrm{HC}$ and PC groups, also with regard to follicular helper T-cells (Fig. 2).

Conversely, striking differences in cell proportions were found in the B-cell compartment, with a marked increase in terminally differentiated cells in COV patients, irrespective of COVID-19 severity, compared to the PC group, and, even more markedly, to the HC group. The increase in terminally differentiated cells resulted in a reduced proportion of memory cells in the COV group compared to both PC and $\mathrm{HC}$ control groups.

\section{Comparison of immunophenotyping characteristics between dialysis and kidney transplant patients and longitudinal analysis}

Comparison of immunophenotyping at baseline between the dialysis and the kidney transplant groups is shown in Fig. 3 and Supplementary Fig. 4. Lower NK and pDC counts, as well as lower levels of monocyte HLA-DR expression were detected in the kidney transplant group, both in the overall cohort and in the NegCOV subgroup. Furthermore, transplant recipients showed higher proportions of switched memory B cells.

For a subset of 13 patients, immunophenotyping was performed at two or more time points (Fig. 4). This analysis included 4 dialysis patients (2 PosCov and $2 \mathrm{NegCov}$ ) and 9 kidney transplant recipients (5 PosCov and 4 NegCov). All the main immune cell populations, as well as monocyte HLA-DR expression, showed a trend to recover over time towards the levels observed in PCs. When comparing temporal trends between NegCOV and PosCOV patients, the rate of recovery appeared higher in the NegCOV group, that had more pronounced cytopenias at baseline, for all parameters except NK cell count. However, none of these comparisons reached statistical significance (Fig. 4).

\section{Levels of soluble inflammatory mediators}

Plasma levels of several cytokines and chemokines were measured at baseline in $10 \mathrm{COV}$ patients (Fig. 5 and Supplementary Fig. 5) and in 7 PCs. The analysis included 4 dialysis patients (2 PosCov and $2 \mathrm{NegCov}$ ) and 6 kidney transplant recipients (3 PosCov and $3 \mathrm{NegCov}$ ). The COV group showed higher levels of CXCL10, CCL2, IL10, IL6, CXCL8, and IFN- $\alpha$, as compared to PCs; these differences were statistically significant for all except IL10 and IL6. For all these cytokines and chemokines, a consistently higher trend was observed in patients with more severe disease (NegCOV). No noticeable changes in the levels of CXCL9, CCL5, TNF- $\alpha$, IL17A, IL4, IL1ß, IL5 and IL12 were detected.

\section{SARS-CoV-2 serology}

Levels of anti-S antibodies were higher in the dialysis population compared to kidney transplant recipients for up to 60 days from symptom onset $(p=0.047)$; the trend was maintained even after adjusting the model for disease severity $(p=0.052)$ (Fig. 6). Anti-N antibodies tended to be lower in kidney transplant recipients as well, although this did not reach statistical significance ( $p=0.143$ ) (Fig. 6). 

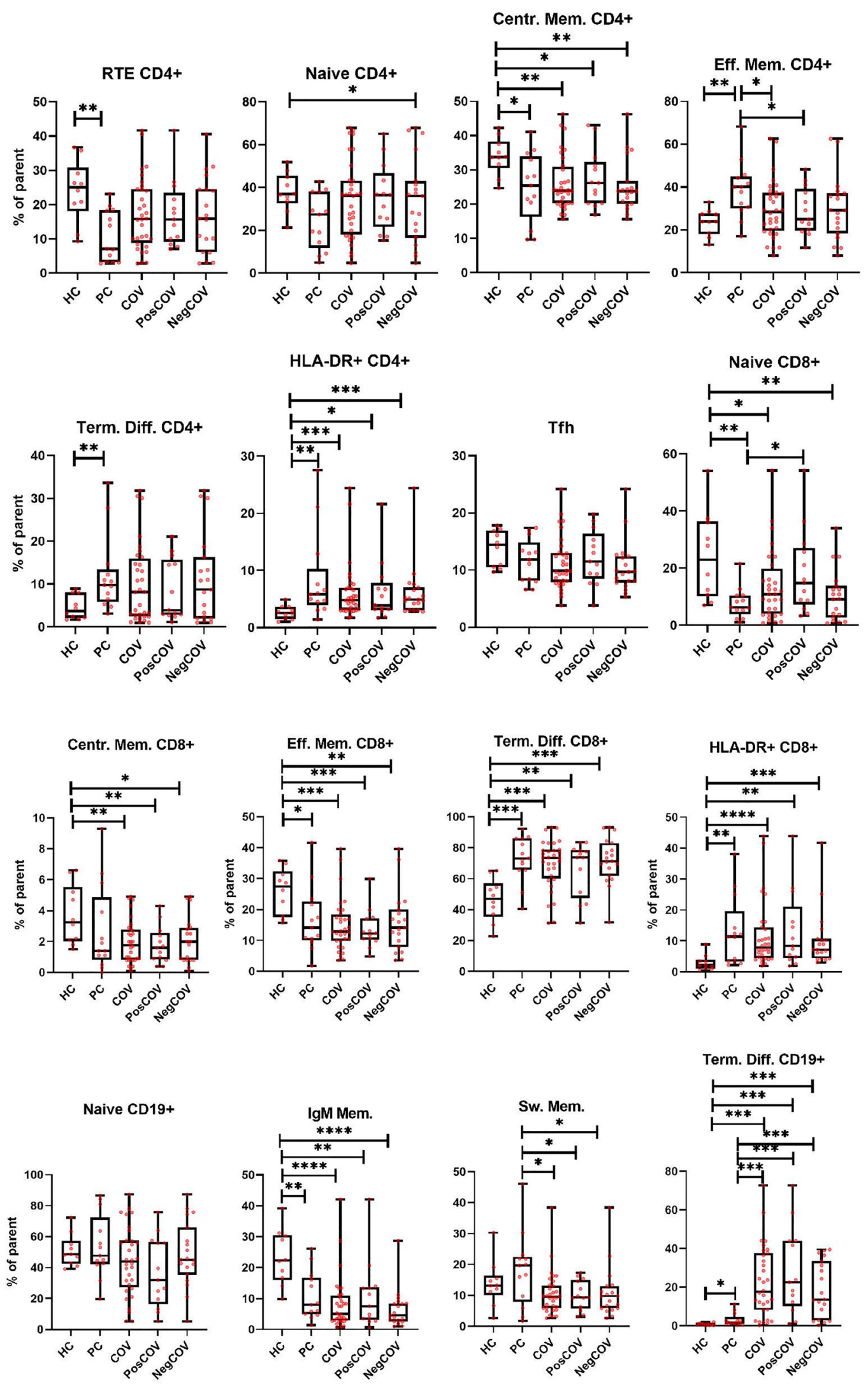
4Fig. 2 Lymphocyte subpopulation distribution at baseline. Comparisons are made across five groups: healthy controls (HC), controls on dialysis or with kidney transplant without SARS-CoV-2 infection (PC), the overall dialysis and kidney transplant population with SARS-CoV-2 infection (COV), the subgroup with SARS-CoV-2 infection and positive outcome (PosCOV) and the subgroup with SARS-CoV-2 infection and negative outcome (NegCOV). The lines inside the boxes represent the median level, the edge of the boxes the 25th-75th percentiles, the whiskers the minimum and maximal values. RTE recent thymic emigrants, Centr. Mem. central memory, Eff. Mem effector memory, Term. Diff. terminally differentiated, Tfh follicular helper T-cells, IgM Mem. IgM Memory B-cells, Sw. Mem. switched memory B-cells

\section{T lymphocytes}
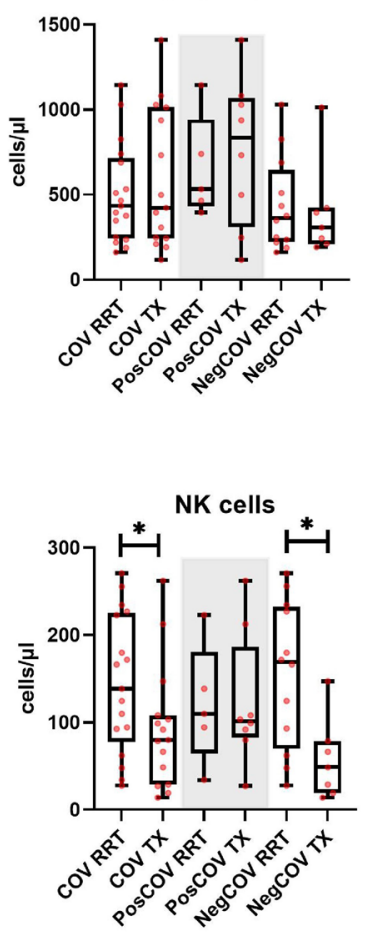

Naive CD19+

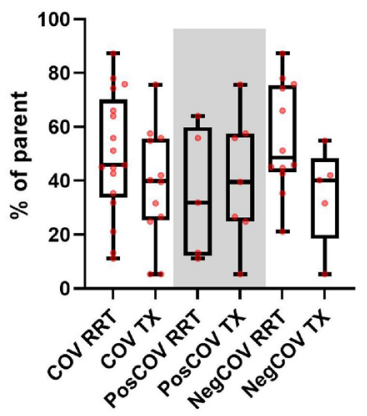

Helper T-cells

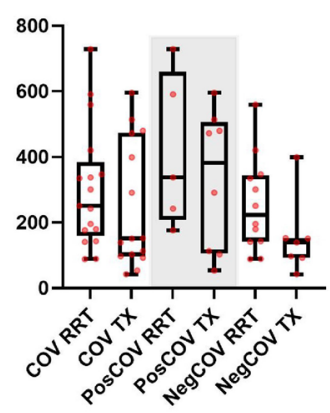

pDC

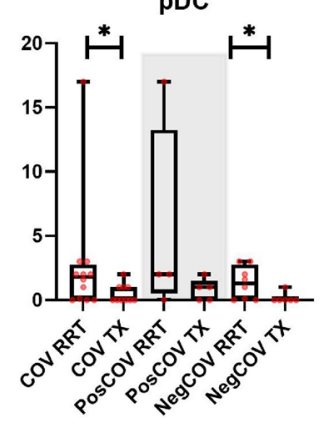

IgM Mem.

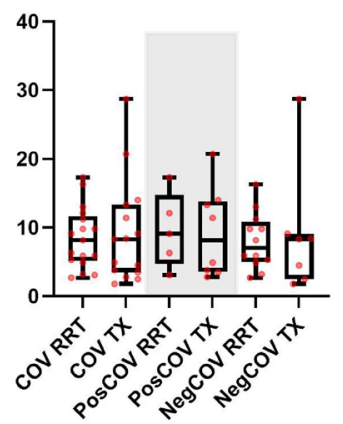

Fig. 3 Comparison of immunophenotyping features at baseline between dialysis and kidney transplant patients with SARS-CoV-2 infection. The lines inside the boxes represent the median level, the edge of the boxes the 25th-75th percentiles, the whiskers the minimum and maximal values. The grey area includes patients belong-

\section{Discussion}

Infection with SARS-CoV-2 can lead to a wide spectrum of clinical phenotypes and outcomes, ranging from asymptomatic infection to life-threatening disease with multi-organ failure. Individual differences in immune response are emerging as key factors in modulating disease severity [30]. Patients on dialysis, and kidney transplant recipients, are at higher risk of severe COVID-19 [31], and a delayed viral clearance has been observed in these patients as well [32]. Of interest, in these groups of frail patients, baseline clinical characteristics cannot effectively predict the evolution of
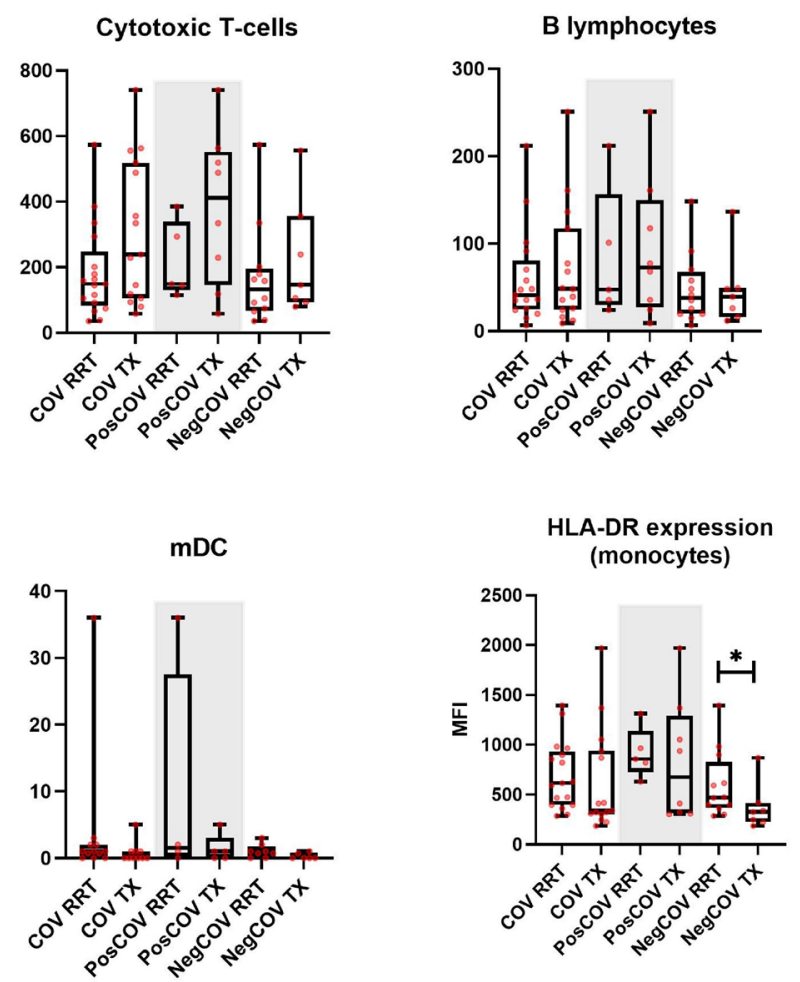

Sw. Mem.

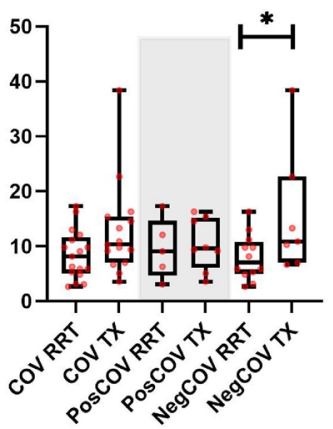

Term. Diff. CD19+

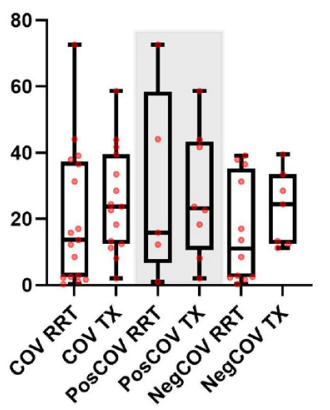

ing to the PosCOV group. $R R T$ renal replacement therapy, $T X$ kidney transplant, $M F I$ mean fluorescence intensity, $p D C$ plasmacytoid dendritic cells, $m D C$ myeloid dendritic cells, IgM Mem. IgM Memory B-cells, Sw. Mem. switched memory B-cells, Term. Diff. terminally differentiated. $* p<0.05$ 

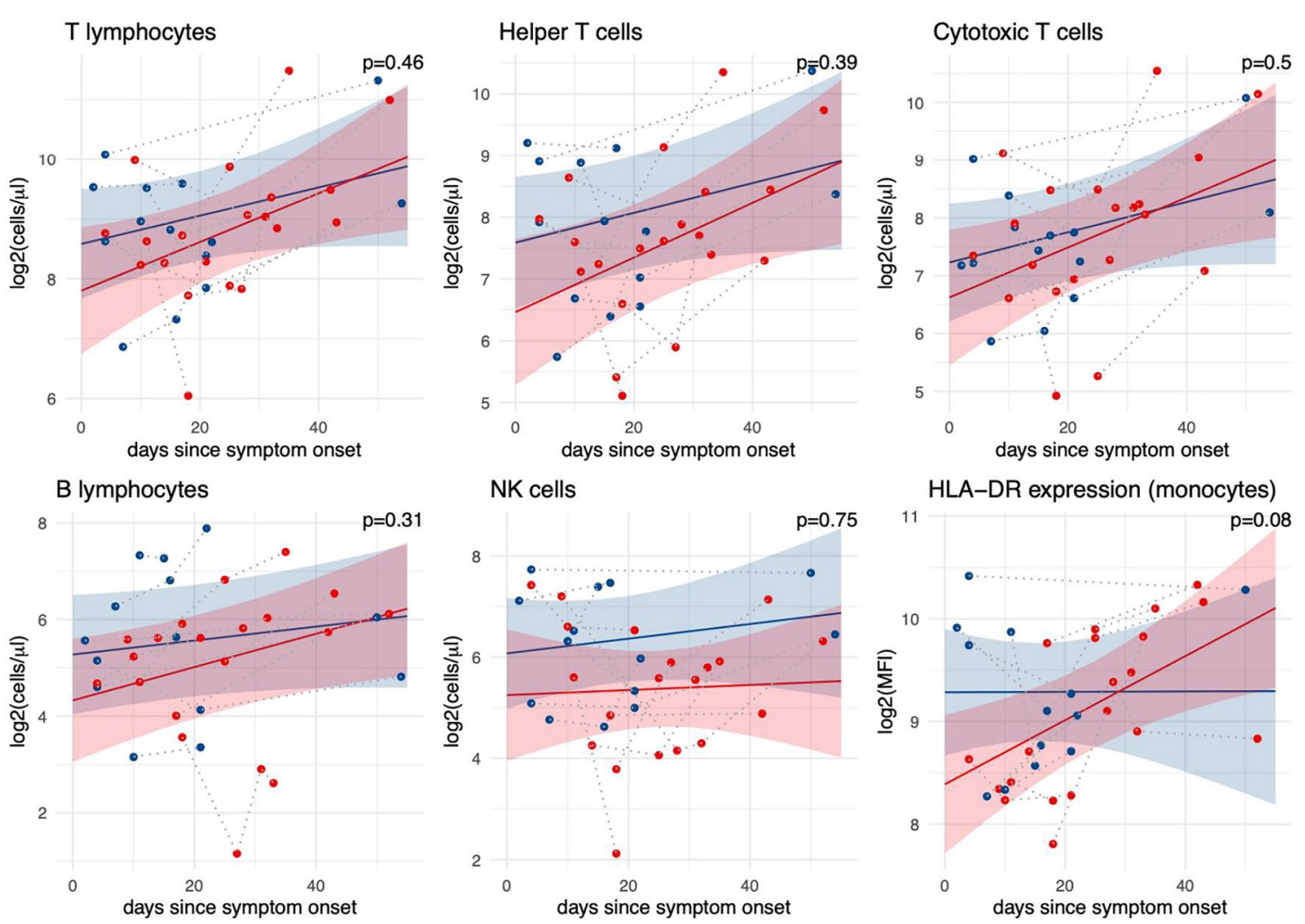

group $\rightarrow$ Poscov $\rightarrow$ NegCOV

Fig. 4 Longitudinal course of cell counts and monocyte HLA-DR expression in a subgroup of 13 patients with SARS-CoV-2 infection. The changes over time of immune parameters were analyzed using linear mixed models. Dots represent individual data points, with grey dotted lines connecting observations from the same patients. Regression lines show trends for COVID-19 patients with positive (PosCOV, blue) or negative outcome (NegCOV,red); ribbons indicate $95 \%$ con-

SARS-CoV-2 infection: in the cohort presented in this study the proportion of patients with bilateral infiltrates at chest $\mathrm{X}$-ray and shortness of breath was similar in the PosCOV and NegCOV groups, with high temperature more often detected in the latter group (although the difference was not statistically significant) and cough reported less frequently in the same subgroup. While the reasons for this are unclear, the dissociation between baseline characteristics and disease evolution has already been described in cohorts of kidney transplant and hemodialysis patients $[15,16]$, further reinforcing the need for biomarker discovery in this context.

However, these groups are usually underrepresented in terms of biomarker and prospective interventional studies. Moreover, data on changes in lymphocyte subsets during SARS-CoV-2 infection occurring in dialysis and kidney transplant patients are scanty, with only two small cohorts published so far [23, 24] that included 7 and 18 patients, respectively. fidence intervals. The reported p-values refer to the statistical significance of the interaction term between outcome group (PosCOV/ NegCOV) and time (days from onset of COVID-19 symptoms). MFI mean fluorescence intensity. The analysis included 4 dialysis patients (2 PosCov and $2 \mathrm{NegCov}$ ) and 9 kidney transplant patients (5 PosCov and 4 NegCov)

In this manuscript, we have focused on several aspects of the immunology of SARS-CoV-2 infection in kidney transplant and dialysis patients. We demonstrated that these groups have significant pre-existing alterations of innate and adaptive immunity, that are further amplified by SARSCoV-2 infection. We also observed that dialysis and kidney transplant patients have overlapping immunophenotyping characteristics, with more evident innate immune defects in kidney transplant recipients.

Our data showed that the immunological profile of patients with kidney disease without COVID-19 (PCs) is indeed significantly different from that of healthy controls, thus reinforcing the importance of including an appropriate control group in the study design to inform a proper interpretation of the results. Uninfected patients with kidney disease displayed reduced counts of all the main circulating lymphocyte populations and of pDCs. Marked differences were observed also within lymphocyte subsets, with a higher 

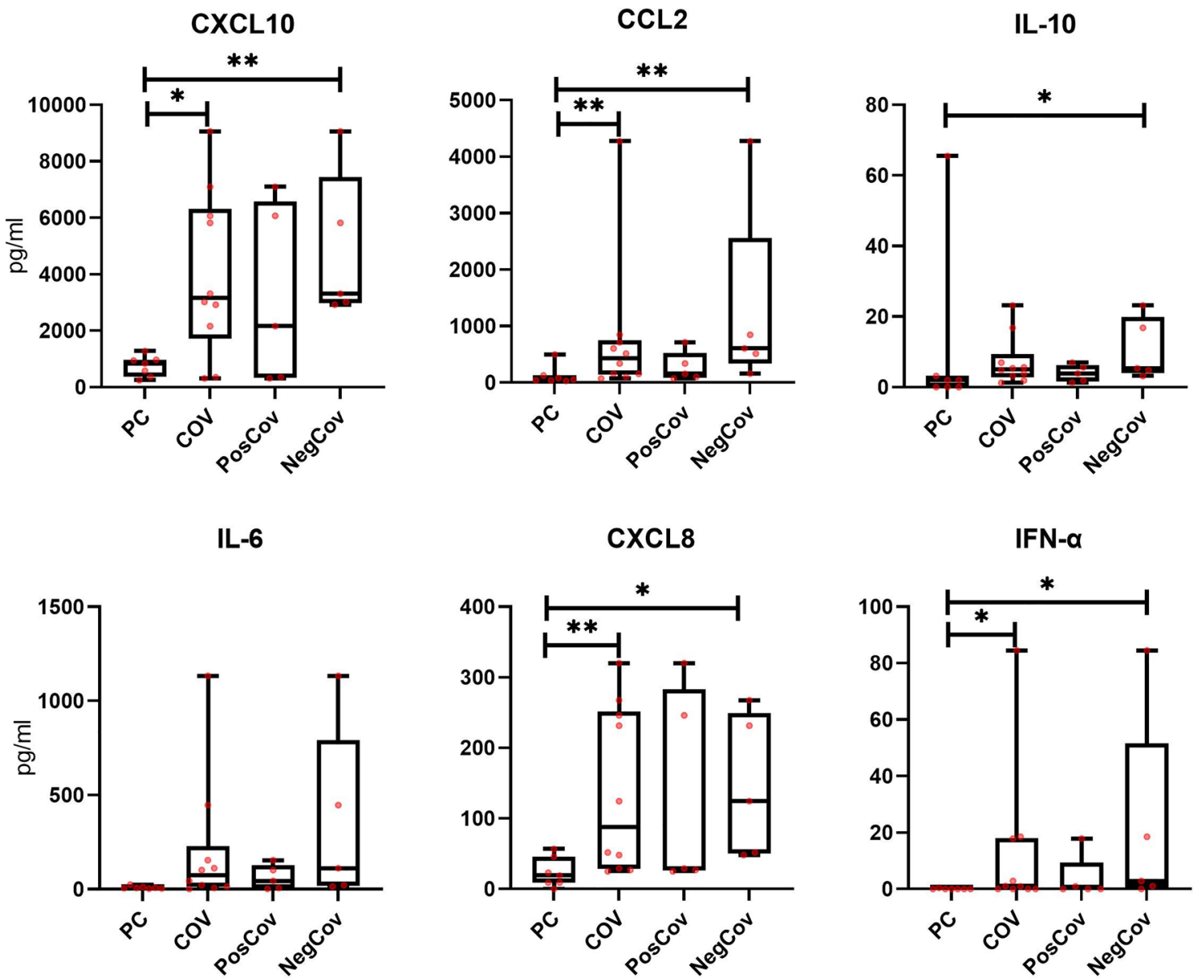

Fig. 5 Cytokines and chemokines at baseline in a subset of 10 dialysis and kidney transplant patients with SARS-CoV-2 infection and 7 pathological controls. Comparisons are made across four groups: controls on dialysis or with kidney transplant without SARS-CoV-2 infection (PC), the overall dialysis and kidney transplant population with SARS-CoV-2 infection (COV), the subgroup with SARS-CoV-2

proportion of terminally differentiated lymphocytes in both the $\mathrm{T}$ and $\mathrm{B}$ cell compartments and a higher percentage of activated HLA-DR $+\mathrm{T}$ lymphocytes in patients with kidney disease.

A limitation of our study is that the HC group was not age-matched to the PC group, being on average younger. Despite that, our observations are in line with previous studies, that also reported an immune signature of lymphopenia with decreased thymic output, increased chronic T-cell activation and reduced numbers of pDCs in both dialysis patients and transplant recipients [18]. These immunological changes closely resemble those of healthy elderly individuals [19], suggesting that accelerated immune senescence in renal patients may contribute to the increased risk of severe COVID-19 in patients with kidney disease, although prospective data are needed to definitely prove this hypothesis. Recent studies identified genetic and immune-mediated infection and positive outcome (PosCOV) and the subgroup with SARS-CoV-2 infection and negative outcome (NegCOV). The lines inside the boxes represent the median level, the edge of the boxes the 25th-75th percentiles, the whiskers the minimum and maximal values. The analysis included 4 dialysis patients (2 PosCov and 2 Neg$\mathrm{Cov}$ ) and 6 kidney transplant patients (3 PosCov and $3 \mathrm{NegCov}$ )

defects in the type I interferon pathway as crucial factors predisposing to severe COVID-19 [33, 34]. pDCs are the main cellular sources of type I interferons, which play a key role in orchestrating the initial immune response to viral infections. It is therefore tempting to speculate that the reduced number of pDCs observed in immune aging may be a particularly important modifier of disease severity in COVID-19, both in the general old population and in patients with kidney disease.

Importantly, in addition to these alterations of the immune landscape that characterize patients with kidney disease, those who developed SARS-CoV-2 infection (COV group) showed further reductions in the counts of all lymphocyte subsets, pDCs and mDCs. The cellular composition of the CD4 + and CD8 + T lymphocyte compartments remained substantially stable during the course of COVID-19, while a pronounced increase in the proportion of terminally 
Fig. 6 Anti-spike and antinucleocapsid protein antibodies over time in 32 dialysis and kidney transplant patients with SARS-CoV-2 infection. Regression lines with $95 \%$ confidence intervals from linear mixed models with quadratic time trend for anti-S (top row) and anti-N (bottom row) antibody levels in the first 60 days following onset of COVID-19 symptoms. The comparison between dialysis (green line) and kidney transplant (violet line), adjusted for COVID-19 severity, is showed in the left panel ( $p$ values in the top right corner). The right panel shows the comparison between Pos$\mathrm{COV}$ (blue line) and $\mathrm{NegCov}$ (red line), adjusted for dialysis/ transplant status. Individual observations are showed as dots, with grey dotted lines connecting data points from the same patients. Antibody levels are expressed as $\log 2$ transformed light units (LU). The horizontal dashed line represents the cutoff for seroconversion. Anti-S antispike protein antibodies, Anti-N anti-nucleocapsid protein antibodies

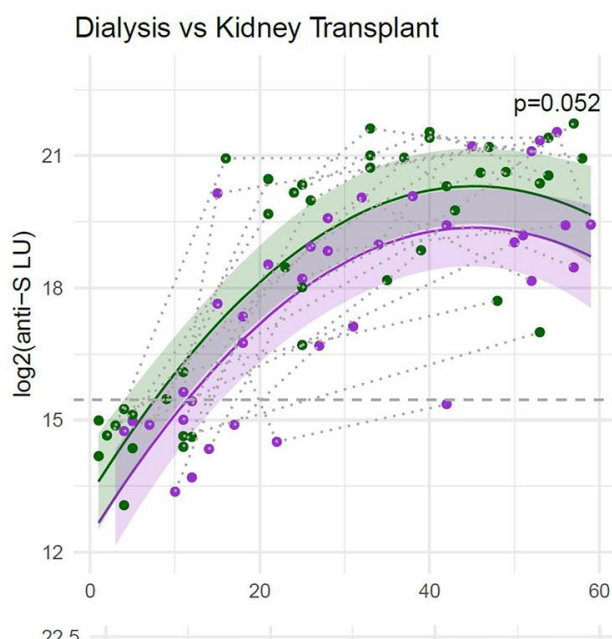

PosCov vs NegCov

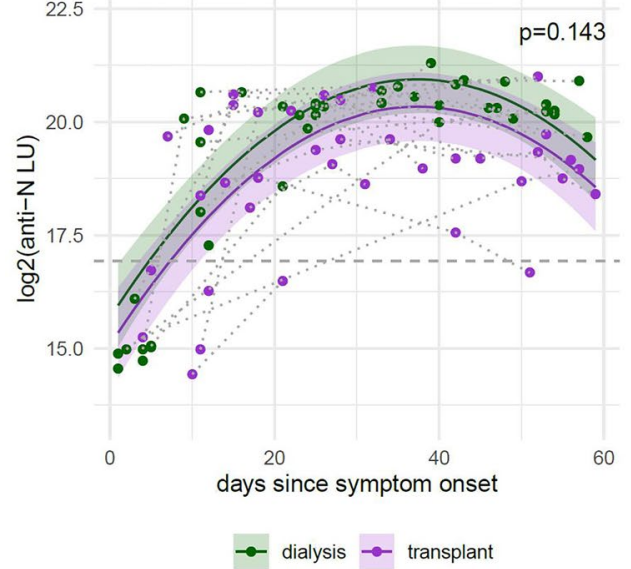

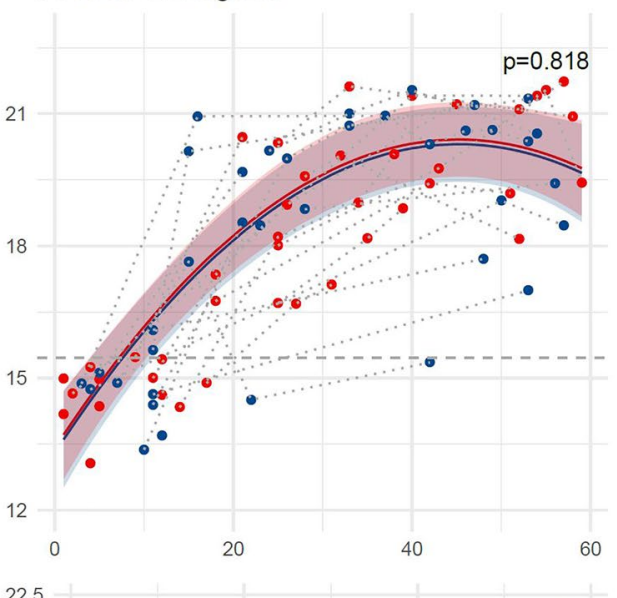
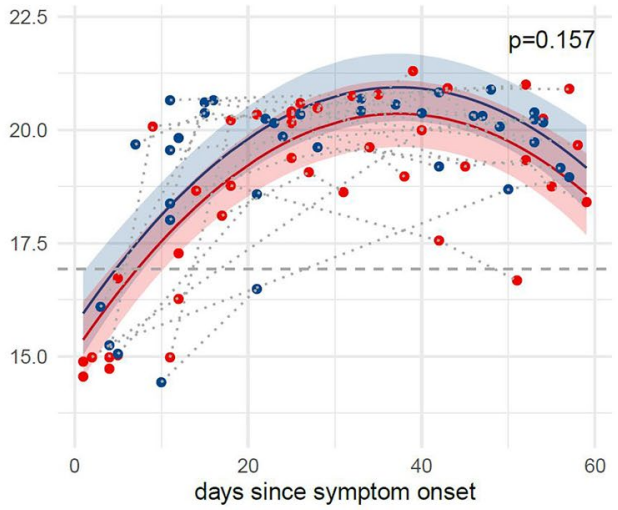

PosCOV $\rightarrow$ NegCOV differentiated cells was observed in the B cell compartment. These changes largely reflect previous observations made in the general population with moderate-severe COVID-19, in terms of both lymphopenia and reduction of circulating DCs, as well as a strong drive on B-cell differentiation [5, $6,8]$. Unlike reports in the general population $[5,12]$, we found that the proportion of activated T-cells, despite being increased at baseline in renal patients, did not undergo a further increase in patients with COVID-19, at least in the first days after the onset of symptoms. We may therefore hypothesize that impaired activation of T cells after SARS-CoV-2 infection, along with chronic abnormal immune stimulation, may further contribute to severe outcome of COVID-19 in patients with kidney disease.

To the best of our knowledge, our study is the first to compare the immunological profile between dialysis and kidney transplant patients during a viral infection. Our data indicate that these groups have a similar distribution of lymphocyte subsets, with few important exceptions. In particular, kidney transplant recipients in the NegCOV group showed lower numbers of NK and pDCs and reduced monocyte HLA-DR expression, when compared to the same group on dialysis. These findings are suggestive of a more profound depression of innate immunity in the NegCOV group with kidney transplant. Immunosuppression may play a role in this: it has been described that calcineurin inhibitors (CNIs) suppress toll-like receptor-dependent activation of monocytes/macrophages [35], and also inhibit NK proliferation [36]. Similarly, mycophenolate mofetil (MMF) may hinder monocyte activation [37, 38], as well as NK proliferation and cytotoxicity [39].

The second difference consists of a higher proportion of switched memory B-cells, in the context of a trend towards a lower proportion of naïve CD19+B-cells and a higher percentage of terminally differentiated B-cells in kidney transplant recipients belonging to the NegCOV group. This finding should be interpreted cautiously, especially in the context of the observation of lower levels of antibodies directed against SARS-CoV-2 in transplant recipients, compared to the dialysis group. If confirmed, these apparently contradictory observations may raise the possibility that immunosuppression in transplant recipients interferes with the late stages of B cell differentiation and IgG secretion. In particular, as supported by in vitro experimental evidence [40] this may be the case with glucocorticoids, to 
which patients in the kidney transplant cohort were chronically exposed.

Of note, SARS-CoV-2 infection in kidney transplant recipients has not been associated with an increased risk of rejection [41]; this is what we observed also in this cohort, where only one patient showed worsening of pre-existing humoral rejection during SARS-CoV-2 infection. No further episodes were observed within 6 months from COVID-19 diagnosis in the remaining patients (data not shown). Interestingly, this happened despite immunosuppression being usually reduced during the acute phase of the disease. Our finding of defects in both T-cell and B-cell compartments during infection may provide, at least to some extent, an explanation for that.

Our study supports the potential role of immunophenotyping and inflammatory cytokines as biomarkers of SARSCoV-2 infection. DCs and, to a lesser extent, NK cell counts were depressed in COVID-19 patients, with no marked differences among COVID-19 severity groups. On the other hand, T and B lymphopenia was evident in the NegCOV group, with cell counts in PosCOV patients similar to the PC group. Reduced monocyte HLA-DR expression also consistently correlated with disease severity, in line with observations in the general population [28].

Longitudinal changes in the main lymphocyte populations and in monocyte HLA-DR expression showed a general trend towards normalization over time, highlighting that timing of testing is a key parameter to take into account when interpreting immune features in this dynamic setting. No significant differences in longitudinal trends between the PosCOV and NegCOV groups were detected, although low statistical power due to the small sample size constitutes a significant limitation.

In keeping with previous reports [11, 42], plasma levels of various pro-inflammatory cytokines (IL6, IFN- $\alpha$ ), antiinflammatory mediators (IL10) and chemokines mediating monocyte and neutrophil recruitment (CXCL10, CCL2, CXCL8) were higher in the COV group compared to the PC group. A trend for further increases was observed in the NegCOV group, suggesting a possible prognostic role for these mediators in renal patients during SARS-CoV-2 infection. Our results did not show a significant increase in TNF- $\alpha$ levels in the COV groups as compared to the PCs. This is in apparent contrast with previous reports in the general population [11]. We may hypothesize that CNIs, which are known to reduce TNF- $\alpha$ secretion [43], could explain, at least in part, our findings.

Limitations of this study need to be acknowledged. Although this is the largest study on the topic performed so far in dialysis and kidney transplant patients, the sample size is still relatively small, especially when moving the focus from the overall cohort to the different subgroups. Furthermore, it has to be underlined that we have included only patients who were followed during the first pandemic wave; of note, at that stage tracking systems and diagnostic tools were scarce and the clinical aspects of the disease still largely unknown. We cannot rule out that these aspects may have had a role, at least in a subset of patients, on the timing of the diagnosis and therefore on disease severity. Moreover, the study groups are heterogeneous under different aspects that contribute to the shaping of the immune landscape, such as gender, age, immunosuppression, dialysis vintage and time since transplant.

We cannot exclude that these factors somehow confounded our findings, which will require validation in bigger cohorts.

The main strength of our work is that it is the first study to address several immunological aspects of COVID-19 in dialysis and kidney transplant in the same cohort, avoiding uncertainties derived from comparisons across different patient cohorts.

In conclusion, patients on dialysis or with kidney transplant have profound baseline immunophenotypic alterations that are further amplified by SARS-CoV-2 infection, especially in the severe forms. Although dialysis and kidney transplant patients are inherently heterogeneous groups, the immunological abnormalities during COVID-19 are similar across the two cohorts, with the exception of more pronounced defects in innate immunity and a dampened antibody response in kidney transplant recipients.

Supplementary Information The online version contains supplementary material available at https://doi.org/10.1007/s40620-021-01214-8.

Acknowledgements The Authors would like to thank Luisa Imberti and Kelvin Hunter for their significant suggestions and contribution in manuscript drafting and revision.

Funding This work has been supported in part by the Division of Intramural Research, National Institute of Allergy and Infectious Diseases and National Institute of Dental and Craniofacial Research, National Institutes of Health.

\section{Declarations}

Conflict of interest FA, consultancy fees from Baxter, AstraZeneca and Otsuka, advisory board for Trevere Therapeutics; AMR research funding from AstraZeneca, European Hematology Association, Transcan2-ERANET, Italian Association for Cancer Research (Fondazione AIRC); advisory board for from Amgen, Celgene, Takeda, Janssen.

Ethical statement The study was approved by the ethical committee of the ASST Spedali Civili Hospital (protocol NP4027).

\section{References}

1. Wu C, Chen X, Cai Y, Xia J, Zhou X, Xu S et al (2020) Risk factors associated with acute respiratory distress syndrome and death 
in patients with Coronavirus Disease 2019 pneumonia in Wuhan, China. JAMA Intern Med 180(7):934

2. Chen G, Wu D, Guo W, Cao Y, Huang D, Wang H et al (2020) Clinical and immunological features of severe and moderate coronavirus disease 2019. J Clin Invest 130(5):2620-2629

3. Diao B, Wang C, Tan Y, Chen X, Liu Y, Ning L et al (2020) Reduction and functional exhaustion of $\mathrm{T}$ cells in patients with Coronavirus Disease 2019 (COVID-19). Front Immunol 1:1. https://doi.org/10.3389/fimmu.2020.00827/full

4. Zhang X, Tan Y, Ling Y, Lu G, Liu F, Yi Z et al (2020) Viral and host factors related to the clinical outcome of COVID-19. Nature 583(7816):437-440

5. Bergamaschi L, Mescia F, Turner L, Hanson AL, Kotagiri P, Dunmore BJ, et al. (2021) Longitudinal analysis reveals that delayed bystander CD8 $+\mathrm{T}$ cell activation and early immune pathology distinguish severe COVID-19 from mild disease. Immunity [Internet]. Available from: https://linkinghub.elsevier.com/retrieve/pii/ S1074761321002168. Accessed 2021 Jun 5

6. Mathew D, Giles JR, Baxter AE, Oldridge DA, Greenplate AR, Wu JE et al (2020) Deep immune profiling of COVID-19 patients reveals distinct immunotypes with therapeutic implications. Science 369(6508):eabc8511

7. Laing AG, Lorenc A, Molino del Barrio I, Das A, Fish M, Monin L et al (2020) A dynamic COVID-19 immune signature includes associations with poor prognosis. Nat Med 26(10):1623-1635

8. Burbelo PD, Riedo FX, Morishima C, Rawlings S, Smith D, Das S et al (2020) Sensitivity in detection of antibodies to nucleocapsid and spike proteins of severe acute respiratory syndrome Coronavirus 2 in patients with Coronavirus Disease 2019. J Infect Dis 222(2):206-213

9. Abers MS, Delmonte OM, Ricotta EE, Fintzi J, Fink DL, de Jesus AAA, et al. (2021) An immune-based biomarker signature is associated with mortality in COVID-19 patients. JCI Insight [Internet]. 6(1). Available from: https://insight.jci.org/articles/view/144455. Accessed 2021 Jun 5

10. Yale IMPACT Team, Lucas C, Wong P, Klein J, Castro TBR, Silva J et al (2020) Longitudinal analyses reveal immunological misfiring in severe COVID-19. Nature 584(7821):463-469

11. Del Valle DM, Kim-Schulze S, Huang H-H, Beckmann ND, Nirenberg S, Wang B et al (2020) An inflammatory cytokine signature predicts COVID-19 severity and survival. Nat Med 26(10):1636-1643

12. Arunachalam PS, Wimmers F, Mok CKP, Perera RAPM, Scott M, Hagan $T$ et al (2020) Systems biological assessment of immunity to mild versus severe COVID-19 infection in humans. Science 369(6508):1210-1220

13. Bossini N, Alberici F, Delbarba E, Valerio F, Manenti C, Possenti S et al (2020) Kidney transplant patients with SARS-CoV-2 infection: the Brescia renal COVID task force experience. Am J Transplant 20(11):3019-3029

14. Alberici F, Delbarba E, Manenti C, Econimo L, Valerio F, Pola A et al (2020) A report from the Brescia Renal COVID task force on the clinical characteristics and short-term outcome of hemodialysis patients with SARS-CoV-2 infection. Kidney Int 98(1):20-26

15. Alberici F, Delbarba E, Manenti C, Econimo L, Valerio F, Pola A et al (2020) A single center observational study of the clinical characteristics and short-term outcome of 20 kidney transplant patients admitted for SARS-CoV2 pneumonia. Kidney Int 97(6): 1083-1088

16. De Meester J, De Bacquer D, Naesens M, Meijers B, Couttenye MM, De Vriese AS et al (2021) Incidence, characteristics, and outcome of COVID-19 in adults on kidney replacement therapy: a regionwide registry study. J Am Soc Nephrol 32(2):385-396

17. Coll E, Fernández-Ruiz M, Sánchez-Álvarez JE, MartínezFernández JR, Crespo M, Gayoso J et al (2021) COVID-19 in transplant recipients: the Spanish experience. Am J Transplant 21(5):1825-1837

18. Betjes MGH (2013) Immune cell dysfunction and inflammation in end-stage renal disease. Nat Rev Nephrol 9(5):255-265

19. Betjes MGH, Langerak AW, van der Spek A, de Wit EA, Litjens NHR (2011) Premature aging of circulating T cells in patients with end-stage renal disease. Kidney Int 80(2):208-217

20. Hutchinson P, Chadban SJ, Atkins RC, Holdsworth SR (2003) Laboratory assessment of immune function in renal transplant patients. Nephrol Dial Transplant 18(5):983-989

21. Kim KW, Chung BH, Jeon EJ, Kim B-M, Choi BS, Park CW et al (2012) B cell-associated immune profiles in patients with end-stage renal disease (ESRD). Exp Mol Med 44(8):465

22. Pahl MV, Gollapudi S, Sepassi L, Gollapudi P, Elahimehr R, Vaziri ND (2010) Effect of end-stage renal disease on B-lymphocyte subpopulations, IL-7, BAFF and BAFF receptor expression. Nephrol Dial Transplant 25(1):205-212

23. Hande A, Musabak U, Ayvazoglu Soy EH, Kurt Azap O, Sayin B, Akcay S et al (2020) Incidence and immunologic analysis of Coronavirus Disease (COVID-19) in hemodialysis patients: a single-center experience. Exp Clin Transplant 18(3):275-283

24. Hartzell S, Bin S, Benedetti C, Haverly M, Gallon L, Zaza G et al (2020) Evidence of potent humoral immune activity in COVID-19-infected kidney transplant recipients. Am J Transplant 20(11):3149-3161

25. Rubenfeld GD et al (2012) Acute respiratory distress syndrome: the Berlin definition. JAMA. https://doi.org/10.1001/jama.2012. 5669

26. Alberici F, Delbarba E, Manenti C, Econimo L, Valerio F, Pola A et al (2020) Management of patients on dialysis and with kidney transplantation during the SARS-CoV-2 (COVID-19) pandemic in Brescia, Italy. Kidney Int Rep 5(5):580-585

27. Kuznetsova A, Brockhoff PB, Christensen RHB (2017) lmerTest Package: Tests in Linear Mixed Effects Models. J Stat Softw [Internet]. 82(13). Available from: http://www.jstatsoft.org/v82/ i13/. Accessed 2021 Jun 5

28. Moratto D, Chiarini M, Giustini V, Serana F, Magro P, Roccaro AM et al (2020) Flow cytometry identifies risk factors and dynamic changes in patients with COVID-19. J Clin Immunol 40(7):970-973

29. Giamarellos-Bourboulis EJ, Netea MG, Rovina N, Akinosoglou $\mathrm{K}$, Antoniadou A, Antonakos N et al (2020) Complex immune dysregulation in COVID-19 patients with severe respiratory failure. Cell Host Microbe 27(6):992-1000.e3

30. Brodin P (2021) Immune determinants of COVID-19 disease presentation and severity. Nat Med 27(1):28-33

31. Williamson EJ, Walker AJ, Bhaskaran K, Bacon S, Bates C, Morton CE et al (2020) Factors associated with COVID-19-related death using OpenSAFELY. Nature 584(7821):430-436

32. Bruno PF, Cappuccilli M, Spazzoli A, De Liberali M, Sejdiu B, Napoli M et al (2021) COVID-19 infection: viral clearance and antibody response in dialysis patients and renal transplant recipients. Nephron 26:1-8

33. Bastard P, Rosen LB, Zhang Q, Michailidis E, Hoffmann $\mathrm{H}-\mathrm{H}$, Zhang Y et al (2020) Autoantibodies against type I IFNs in patients with life-threatening COVID-19. Science 370(6515):eabd4585

34. Zhang Q, Bastard P, Liu Z, Le Pen J, Moncada-Velez M, Chen J et al (2020) Inborn errors of type I IFN immunity in patients with life-threatening COVID-19. Science 370(6515):eabd4570

35. Howell J, Sawhney R, Testro A, Skinner N, Gow P, Angus P et al (2013) Cyclosporine and tacrolimus have inhibitory effects on toll-like receptor signaling after liver transplantation: TLR function after liver transplantation. Liver Transpl 19(10):1099-1107

36. Si Y-Q, Bian X-K, Lu N, Jia Y-F, Hou Z-H, Zhang Y (2012) Cyclosporine Induces Up-regulation of Immunoglobulin-like 
Transcripts 3 and 4 expression on and activity of NKL cells. Transplant Proc 44(5):1407-1411

37. Kannegieter NM, Hesselink DA, Dieterich M, Kraaijeveld R, Rowshani AT, Leenen PJM et al (2017) The effect of tacrolimus and mycophenolic acid on CD14+ monocyte activation and function. PLoS ONE 12(1):e0170806

38. čOlić M, Stojić-Vukanić Z, Pavlović B, Jandrić D, Stefanoska I (2003) Mycophenolate mofetil inhibits differentiation, maturation and allostimulatory function of human monocyte-derived dendritic cells: effect of mycophenolate mofetil on human DC. Clin Exp Immunol 134(1):63-69

39. Ohata K, Espinoza JL, Lu X, Kondo Y, Nakao S (2011) Mycophenolic acid inhibits natural killer cell proliferation and cytotoxic function: a possible disadvantage of including mycophenolate mofetil in the graft-versus-host disease prophylaxis regimen. Biol Blood Marrow Transplant 17(2):205-213

40. Palmer L, White RR, Johnson BH, Fowler R, Acosta CJ (2014) Herpes zoster-attributable resource utilization and cost burden in patients with solid organ transplant. Transplantation 97(11):1178-1184
41. Gandolfini I, Zanelli P, Palmisano A, Salvetti D, Parmigiani A, Maltzman JS et al (2021) Anti-HLA and anti-SARS-CoV-2 antibodies in kidney transplant recipients with COVID-19. Transpl Int 34(3):596-599

42. Gisby J, Clarke CL, Medjeral-Thomas N, Malik TH, Papadaki A, Mortimer PM, et al. (2021) Longitudinal proteomic profiling of dialysis patients with COVID-19 reveals markers of severity and predictors of death. eLife [Internet]. Available from: https://elife sciences.org/articles/64827. Accessed 2021 Jun 5

43. Szabo G, Gavala C, Mandrekar P (2001) Tacrolimus and cyclosporine a inhibit allostimulatory capacity and cytokine production of human myeloid dendritic cells. J Investig Med 49(5):442-449

Publisher's Note Springer Nature remains neutral with regard to jurisdictional claims in published maps and institutional affiliations. 